\title{
Astrocytes silence PTEN to promote brain metastasis
}

To initiate metastasis, a disseminated tumour cell must adapt to the local microenvironment, but the signals regulating this process remain unclear. Identifying one such signal, a paper in Nature reports that an astrocyte exosome-derived microRNA induces the loss of PTEN expression in disseminated tumour cells in the brain, thus promoting metastasis.

Analyses of microarray data from both patient samples and mouse models revealed significantly reduced PTEN expression in brain metastases compared with primary breast tumours and metastases in other organs. PTEN loss was also shown by immunohistochemistry to be significantly more common in brain metastases than in both unmatched and matched primary breast cancers.

Although these data suggested a role for PTEN loss in brain metastasis, the authors unexpectedly found that intracarotid injection of PTENknockdown tumour cells did not increase the incidence or size of brain metastases in mice. Furthermore, PTEN levels in the primary tumours of patients with breast cancer were not predictive of the likelihood of brain metastasis-free survival, or of the presence or absence of brain metastases. Together, these data suggested a non-genetic origin for the brain metastasis-associated PTEN loss. Indeed, the authors showed that in vivo brain metastases from single-cell-derived clones expanded from PTEN-normal human breast cancer cell lines exhibited PTEN loss that was not present in lung metastases, suggesting that the PTEN-loss phenotype was induced by the brain microenvironment rather than originating from PTEN-low cells in the primary tumour. Notably, PTEN loss was reversible: PTEN expression in sublines generated from PTENlow brain metastases returned to parental levels in cell culture.

The authors then investigated which cellular component of the microenvironment reversibly induced PTEN loss and demonstrated that tumour cells co-cultured with primary mouse astrocytes showed significantly reduced PTEN expression. Hypothesizing a role for microRNAs in astrocyte-induced PTEN loss, the authors examined brain metastases induced by injecting syngeneic mouse melanoma cells into mice that had an astrocyte-specific deletion of microRNAs known to regulate Pten. In vivo deletion of these microRNAs significantly reduced brain metastasis growth and prevented PTEN loss. Further in vitro studies identified that miR-19a was the specific microRNA responsible for this effect.

The presence of miR-19a and the loss of PTEN were observed in tumour cells cultured in astrocyteconditioned medium. Transmission electron microscopy detected exosomes in the medium that were then shown to contain miR-19a and to induce dose-dependent PTEN loss in tumour cells. Furthermore, inhibition of exosome secretion from astrocytes reduced miR-19a levels in co-cultured tumour cells, rescued PTEN loss both in vitro and in vivo, and significantly reduced brain metastasis outgrowth in mice inoculated with melanoma cells. Notably, in the absence of endogenous astrocyte exosome secretion,

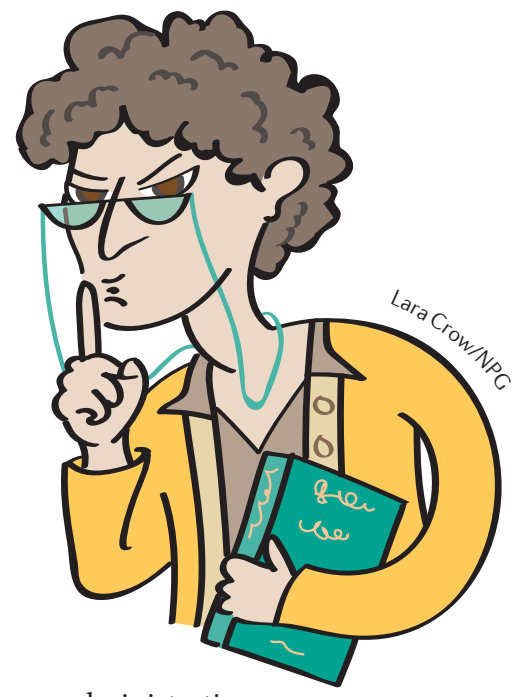

co-administration

of astrocyte-derived exosomes with melanoma cells induced loss of PTEN expression in these cells and promoted brain metastasis in vivo.

Having elucidated the mechanism upstream of PTEN loss, the authors carried out further experiments using mouse models and patient samples to identify the pathway linking PTEN loss to downstream promotion of brain metastasis. Their data implicated nuclear factor- $\kappa \mathrm{B}$ signalling and $\mathrm{C}-\mathrm{C}$ motif chemokine 2 (CCL2)-induced myeloid cell recruitment in this pathway, indicating that there is reciprocal crosstalk between tumour cells and the microenvironment: tumour cell PTEN loss is both induced by and has consequences for the microenvironment.

In summary, this paper reports a brain-metastasis-specific mechanism in which astrocytes induce a reversible loss of PTEN in disseminated tumour cells to prime metastatic outgrowth. It also highlights the role of crosstalk and co-evolution between disseminated tumour cells and the microenvironment in the development of brain metastases.

\section{Lydia Shipman}

ORIGINAL RESEARCH PAPER Zhang, L.,

Zhang, S. et al. Microenvironment-induced PTEN loss by exosomal microRNA primes brain metastasis outgrowth. Nature http://dx.doi. org/10.1038/nature15376 (2015) 